ISSN: 0514-7336

DOI: http://dx.doi.org/10.14201/zephyrus20178093112

\title{
EL ARTESANADO ALFARERO EN IBERIA A FINALES DE LA EDAD DEL HIERRO Y EL INICIO DE LA CONQUISTA ROMANA: CALIDAD VS. CANTIDAD
}

\section{Potter craftsmanship in Iberia at the end of the Iron Age and the beginning of Roman conquest: Quality vs. Quantity}

Juan Jesús Padilla Fernández

Dpto. de Prehistoria. Facultad de Geografía e Historia-UCM. C/ Profesor Aranguren, s/n. Edificio B, Ciudad Universitaria. 28040 Madrid. Correo-e: juanjpad@ucm.es

Recepción: 16/09/2016; Revisión: 23/03/2017; Aceptación: 10/07/2017

RESUMEN: Muchos han sido los trabajos que han intentado abordar la complejidad que subyace en los conjuntos cerámicos elaborados tras la conquista de Roma. Tanto es así que hoy en día es posible encontrar con facilidad un abultado listado de compendios tipológicos que, mayoritariamente, ayudan a fijar secuencias temporales marcadas por una linealidad manifiesta. Una realidad, sin embargo, bien distinta a la que acontece en torno al estudio de las personas encargadas de llevar a cabo su manufactura. Este trabajo pretende, en la medida de lo posible, paliar este vacío de conocimiento, aportando una visión alternativa acerca del artesanado alfarero en los albores de la Edad del Hierro y el impacto que éste sufre como consecuencia del proceso de conquista y aculturación romana. A través de la tecnología y el concepto de Cadena Técnico-Operativa es posible atisbar una serie de cambios y resistencias técnicas que no sólo ayudan a entender las particularidades intrínsecas de las tareas alfareras hace ya dos mil años, sino también la idiosincrasia propia de una sociedad en continua evolución.

Palabras clave: Tecnología cerámica; estatus; individualidad; producción en masa; especialización.

АвSTRACT: There have been many studies that have attempted to address the complexity underlying manufactured ceramic assemblages after the conquest of Rome. So much so that today it is possible to easily find a great amount of typological lists, which mostly help to clearly establish linear temporal sequences. A reality that is most probably very different from the one that really occurred around the people responsible for carrying out their manufacturing. This paper aims, as far as possible, to overcome this lacuna by providing an alternative view about potter craftsmanship at the dawn of the Iron Age, as well as the impact it suffers as a result of the Roman conquest and acculturation. Through technology and the concept of Technical Operational Chain it is possible to glimpse a series of changes and technical resistances, which not only help to understand the intrinsic characteristics of the pottery work that occurred two thousand years ago, but also the idiosyncrasies of a society which is continually evolving.

Key words: Ceramic technology; status; individuality; mass production; specialization.

\section{Introducción ${ }^{1}$}

La arqueología como disciplina siempre ha prestado un especial interés por la cerámica. Esta predilección

1 Un agradecimiento sincero a los profesores G. Ruiz Zapatero, J. R. Álvarez Sanchís y G. Aranda Jiménez que, con sus notas y correcciones, han ayudado a terminar de subyace, esencialmente, en su mayoritaria y frecuente presencia dentro del registro. Los estudios sobre conjuntos cerámicos en España han sido abordados mayoritariamente por las escuelas histórico-cultural

apuntalar todo lo planteado en estas líneas. También quisiera agradecer los comentarios de los evaluadores anónimos querevisaron este artículo y la disposición de A. Rodero Riaza y 
y procesual (p. ej. Bernal y Rivera, 2008, 2012), centrando su discurso analítico en las estrategias tipológicas, con la principal pretensión de crear secuencias o marcos cronoculturales progresivos y unilineales.

No obstante, un pequeńo sector de la academia española comienza a modificar de forma lenta y gradual su visión de la materialidad, concibiéndola no como un conjunto individualizado de entes vacíos, sino como un todo que permite desgranar las múltiples esencias de la cultura a la que pertenece (p. ej. Bermejo, 2010; Sánchez López y Gozalbes, 2012; Mañas y Bermejo, 2013; Gutiérrez Rodríguez et al., 2017); y es que cualquier sujeto o acción acaban siendo condicionados por la realidad para la que han sido preconcebidos (Skibo y Schiffer, 2008). Conocer el modo de manufactura de los objetos nos ayudaría a descifrar parte de los interrogantes que aún tenemos acerca de las comunidades en las que éstos fueron producidos y consumidos. De hecho, numerosos estudios etnográficos y etnoarqueológicos (González Ruibal, 2005; Livingstone, 2007; Gosselain, 2011; Pikirayi y Lindahl, 2013) han demostrado que la cerámica es más que un objeto que sirve para contener líquidos o almacenar productos. Estrechamente ligada a las gentes que la generan, ésta se constituye como una fuente importante de información social (Hodder, 2012). En esencia, puede ser considerada como una herramienta eficaz en la que quedan reflejados códigos y estructuras sociales (Dobres, 2000).

La técnica puede definirse como una parte importante de la cultura (Dietler y Herbich, 1998). Lógicamente, cuando los contextos y la conservación así lo permiten (García Rosselló y Calvo, 2013: 27), los fragmentos cerámicos actúan como contenedores de saber, encerrando en su interior informaciones de todo tipo, circunscritas al ámbito económico, social y simbólico en el que se insertan (Gosselain, 2002). La tecnología está repleta de mensajes con significado y crea bienes materiales en base a determinadas condiciones de vida (Lemonnier, 2012). El concepto de Cadena Técnico-Operativa, definido éste como

E. Manso Martín para poder acceder a determinados materiales arqueológicos conservados en el Museo Arqueológico Nacional y que aquí se citan. De los fallos y errores constatables en el texto, el autor se considera el único responsable. el cómputo de acciones y gestos realizados desde la obtención de la materia prima hasta la configuración del producto final (Balfet, 1975: 52; Creswell, 1976: 13), así como su posterior uso y distribución (Lemonnier, 1993), puede concebirse como la herramienta perfecta con la que poder descifrar dichos mensajes. Su uso se entiende como un eje que conecta simultáneamente el trabajo técnico con entramados socioeconómicos, ideológicos y espaciales (Roux, 2009). Además, la construcción y posterior comparación sistémica de varias Cadenas Operativas suele revelar una serie de cambios o similitudes, que de igual forma afectan a los agentes sociales que participan en su desarrollo (Giddens, 2003). La ruptura o continuidad de determinadas estructuras significaría, por tanto, el mantenimiento, o no, de percepciones concretas de la realidad.

¿Qué papel juega el artesanado alfarero en los últimos compases de la Edad del Hierro y el inicio de época romana? Desde una perspectiva crítica y transversal (Van der Leeuw, 1993; Bowser, 2000), en estas líneas tratamos de abordar un estudio que intenta resolver esta cuestión. Nos hallamos ante un periodo de tránsito, interacción y de gran dinamismo, que deja una profunda huella en la cultura material, y que sin duda debemos aprovechar. Técnicamente, se producen una serie de transformaciones que reflejan en sí mismas un fuerte cambio en la organización económica y social. Las demandas exigidas por un mundo nuevo regido desde Roma cambian las reglas del juego, imponiéndose acusadas modificaciones de índole técnica. A través de una investigación pormenorizada de las Cadenas Técnico-Operativas propias de este periodo, interpretamos la revolución que sufre y experimenta el oficio cerámico, y que, a pesar de lo estipulado por el discurso positivista convencional (Morel, 1991), no supone un avance en calidad y estatus profesional. Precisamente, la necesidad de ahondar en esta comparativa justifica, en primer lugar, un examen pormenorizado de la cerámica prerromana. Explorar los orígenes que asientan el desarrollo de una pauta de manufactura que perdura y se prolonga durante más de siete siglos ayudará a definir todas las modificaciones tecnológicas ligadas al cambio social y económico que promueve la conquista romana. 


\section{El oficio alfarero en la Edad del Hierro: conocimiento y poder}

Gordon Childe (1954), en su famosa obra Rotary Motion, considera la invención del torno como un avance tecnológico en toda regla, precisamente por contribuir a la mejora del trabajo alfarero. Una visión a todas luces funcionalista que, a priori, parece lógica teniendo en cuenta la posibilidad de producir un mayor número de piezas cerámicas en un determinado periodo de tiempo, pero que no concuerda con ciertas informaciones de carácter antropológico y etnoarqueológico (Roux, 2003). Además, en la península ibérica, y a pesar de que tenemos constancia de elementos importados y obtenidos con fuerza cinética en la Edad del Bronce-ss. XIII-XII a. C. (Perlines, 2005)-, no será hasta bien entrado el s. viII a. C. cuando podamos asegurar que son los grupos indígenas, aquellos que negocian con comerciantes procedentes del Mediterráneo, los productores directos de cerámica a torno (Vallejo, 2007). Los datos arqueológicos parecen confirmar que la noción y comprensión de la energía rotatoria por parte de una comunidad, no supondría una modificación inmediata en la Cadena Técnico-Operativa. Toda secuencia de manufactura se caracteriza por ser tremendamente conservadora, rechazando siempre un cambio exógeno, a menos que sea estrictamente necesario (Roux y Corbeta, 1990).

De este modo, y si asumimos lo expuesto como un hecho plausible ¿por qué adquirir un gesto nuevo que rompa con un comportamiento tecnológico específico y comporte un cambio en el modo de fabricar cerámica? Planteándolo sólo como hipótesis de partida, quizás la necesidad de justificar la consolidación de prácticas sociales diferentes ayudaría a entender la incorporación de otras estrategias de producción. Con el objetivo de establecer su propio "régimen de verdad", y así sustentar el poder que las rige (Foucault, 1992: 187), las comunidades de la protohistoria peninsular manipularían su habitus (Bourdieu, 1988: 170-171), ajustándose y adaptándose a las estructuras sociales
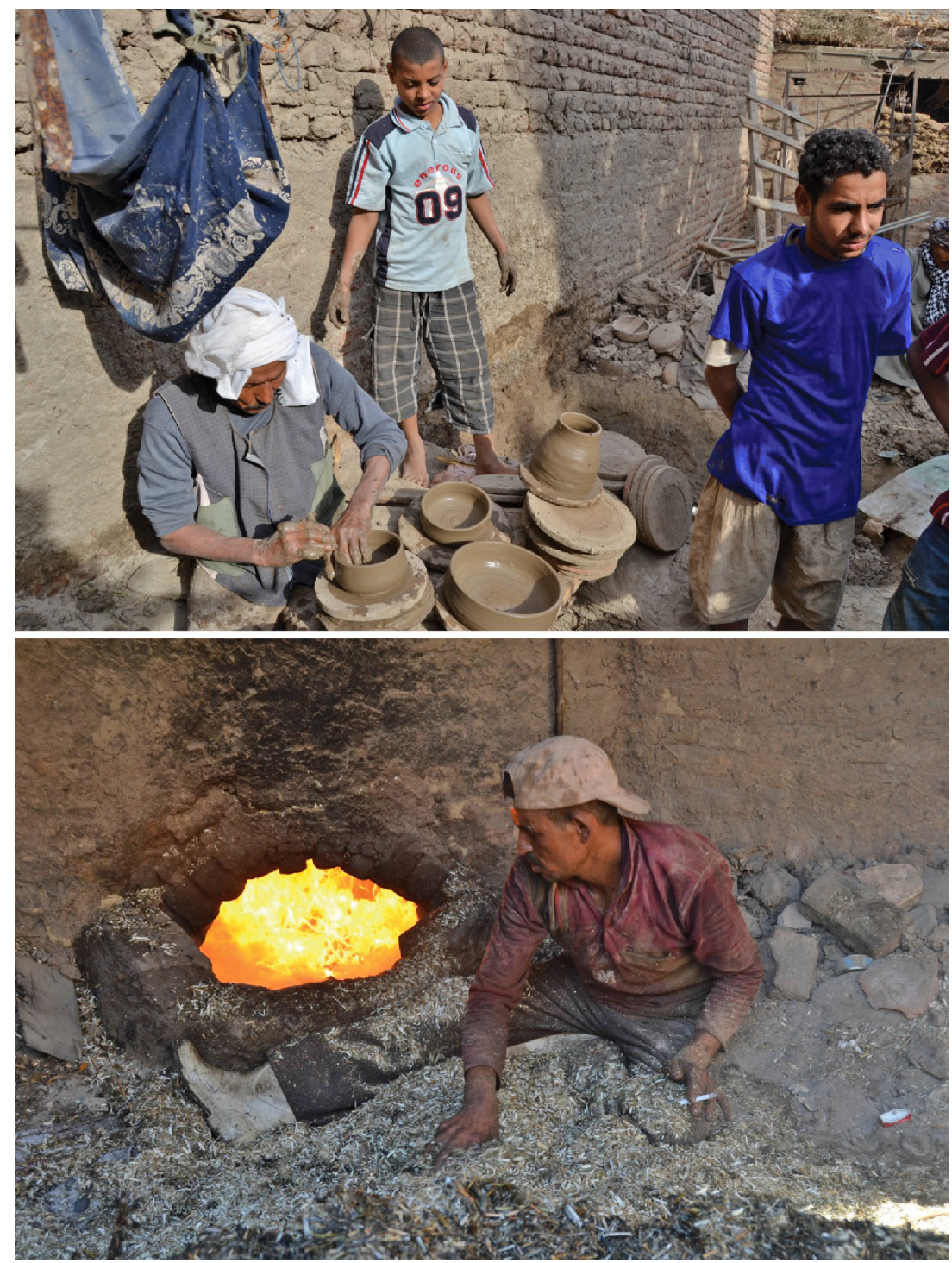

FIG. 1. Maestros alfareros de Hagaza y el-Deir el Gharbi -Luxor, Egipto-modelando y cociendo piezas cerámicas torneadas. 
recién impuestas. Puede que la instauración de contextos sociales cada vez más jerarquizados e individualizados obligara a fijar posiciones de poder en todos los ámbitos de la cultura.

La incorporación del torno alfarero implica la modificación de, al menos, dos fases del proceso manual de fabricación cerámica, el modelado y la cocción, así como una serie de cambios en las recetas de materiales, el modo de organización, de los espacios empleados, el consumo y el propio personal artesano. Es adquirido un modelo productivo que exige un proceso de aprendizaje largo y difícil, requiere una inversión de tiempo igual o superior a 10 años (Gandon et al., 2013) y no comporta mayores ventajas de ejecución que otras técnicas (Roux y Corbeta, 1990), pero, en cambio, sí promueve un control férreo de los medios de producción en ámbitos especializados y ayuda a consolidar una organización social y económica basada en la desigualdad (Roux, 2008; Thér et al., 2017). En consecuencia, quedaría conformado un oficio separado totalmente de lo doméstico, organizado alrededor de una estratificación y autoridad marcada por la sabiduría, la especialización y la experiencia práctica.

La elección tecnológica del torno reforzaría la configuración de una visión determinada del mundo, supeditada a la denominada "fantasía de la individualidad” (Hernando Gonzalo, 2012); una visión del mundo que naturalizaría la imposición de un sistema patriarcal, donde la cerámica a mano, vinculada con la esfera de lo colectivo, sería relegada a un segundo término (Padilla y Chapon, 2015). Una perspectiva que, precisamente, aspira a concienciar sobre la necesidad de asumir el vínculo efectivo existente entre sociedad y cultura material (García Rosselló, 2008), al igual que la diversidad y multiplicidad de dichas conexiones (Lemonnier, 2012).

Una larga lista de aproximaciones etnoarqueológicas y etnográficas parecen mostrar que este automatismo social de la tecnología no es tan descabellado como al principio pudiera parecer (González Ruibal, 2005; Cárdenas y Agudo, 2012; Djorjevic, 2012). De hecho, la posibilidad de poder realizar de primera mano trabajos etnoarqueológicos con comunidades musulmanas alfareras de Luxor-Egipto-ha permitido

(C) Universidad de Salamanca reforzar esta visión aún más si cabe. Éstas organizan el trabajo en torno a dos conceptos: conocimiento y poder. Los grados de habilidad y destreza marcan el desarrollo de cada una de las etapas necesarias para llevar a cabo la manufactura de una cerámica a torno, mostrando especial hincapié en dos de ellas: el modelado y la cocción (Fig. 1).

La aplicación de una herramienta que genera fuerza centrífuga exige la enseñanza pormenorizada de gestos técnicos por parte de los maestros. Individuos infantiles masculinos aprenden, en primer lugar, a pisar y amasar la arcilla ya decantada, una actividad que combinan con la realización de tareas externas y de acarreo. El dominio de tales acciones, otorga el derecho a poder asimilar las llaves o reglas que son esenciales para el torneado. El control y equilibrio, tanto de las posiciones de mano como de la velocidad ejercida, refuerza el dominio activo de los expertos, que en ningún caso es cuestionado.

La cocción también refuerza la consolidación de un sistema productivo estructurado a través de la cualificación. El uso de estructuras bicamerales y de tiro vertical asegura el éxito de una fase crucial y, al mismo tiempo, refuerza los patrones de jerarquización impuestos. Los aprendices alfareros de Luxor tienen prohibida la participación en esta fase, del mismo modo que las mujeres, tachadas de impuras y propiciadoras de dificultar e interrumpir la transformación físico-química de la arcilla en cerámica.

Nos encontramos, efectivamente, ante una serie de espacios a modo de taller, que están regidos por maestros respetados que fabrican de forma sistemática productos que requieren un nivel elevado de pericia técnica. Éstos, y al igual que otros centros diseminados por el territorio egipcio (Cooper, 2000; Duistermaat y Groot, 2008; Nicholson, 2009), suplen la mayor parte de la demanda cerámica generada por la sociedad de religión musulmana. No obstante, en determinadas regiones como Siwa, todavía es posible encontrar, marginalmente, piezas cerámicas que continúan siendo modeladas a mano. El estudio de los procesos de trabajo, así como de la figura de su artesanado (Nicholson, 2009; Calvo Trías y García Rosselló, 2012), evidencia diferencias sustanciales respecto a los documentados para 

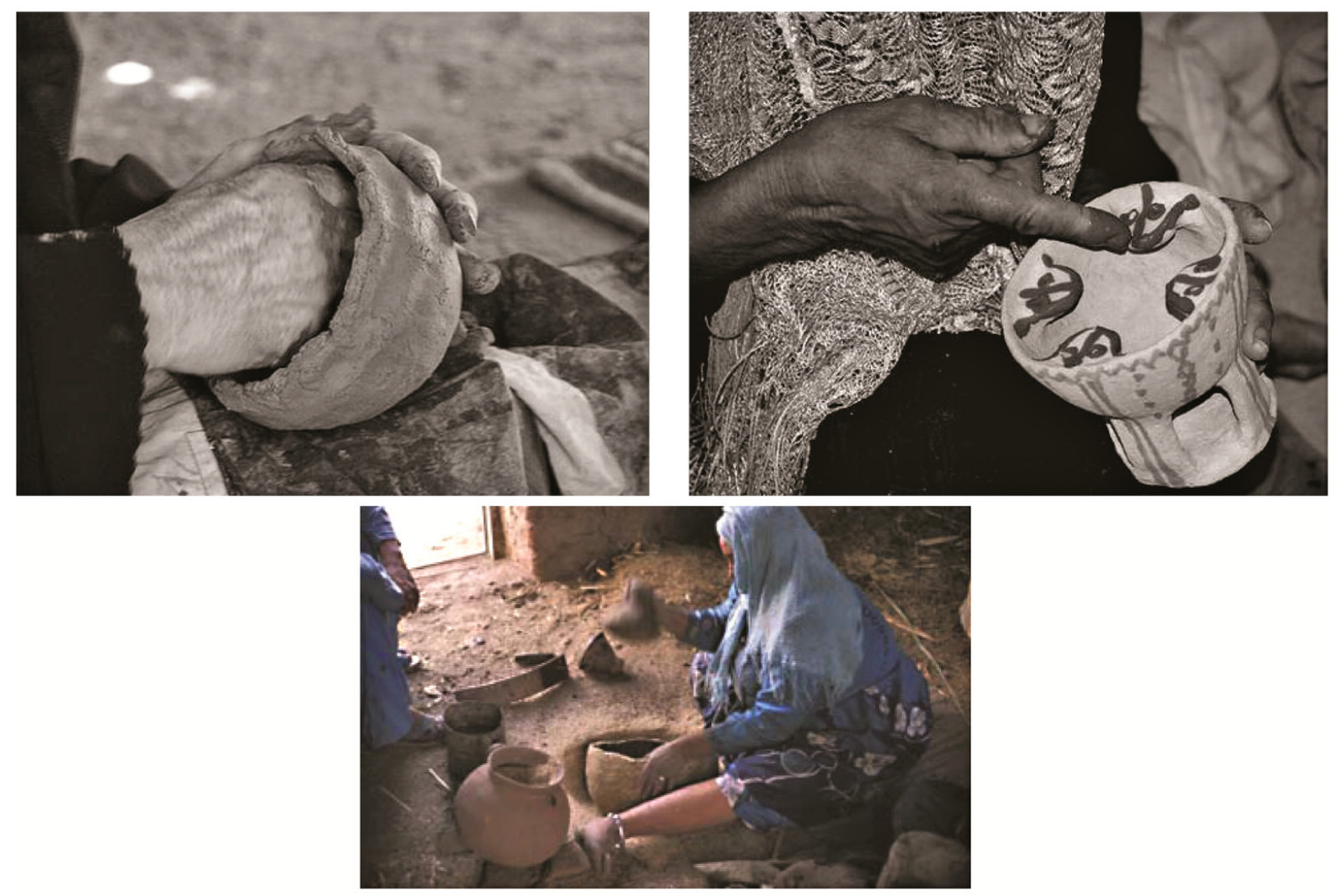

Fig. 2. Maestras alfareras de Siwa y Deir Mawas, Egipto, modelando y decorando manualmente piezas cerámicas (Calvo Trías y García Rosselló, 2012: 397, fig. 1; Nicholson, 2009: 4, fig. 5).

la producción de útiles torneados. Esta actividad alfarera parece ser concebida como una actividad más de mantenimiento y de perfil colectivo, efectuada en su mayoría por mujeres y transmitida por medio de un aprendizaje en el que no manda necesariamente el interés por alcanzar la maestría (Fig. 2).

La cerámica consumida por las comunidades musulmanas egipcias refleja de manera fiel la política patriarcal que condiciona todas y cada una de las esferas de su sociedad. En coherencia con el resto de dimensiones sociales, el control de la tecnología alfarera contribuye al reflejo de la creciente individualidad masculina, al mismo tiempo que asienta diferentes categorías de estratificación jerárquica, posicionando siempre a lo femenino en esferas marginales.

¿Qué ocurriría si la disciplina arqueológica centrara su interés en las relaciones habidas entre el comportamiento humano y la cultura material? $\mathrm{Si}$ actualmente, en comunidades preindustriales como la egipcia, la función tecnológica ejerce al mismo tiempo una función social específica (Schiffer, 1992: 132-133), ¿por qué no pensar en la existencia de una ejecución de roles similares en el pasado? Considerando, lógicamente por encima de todo, que las dinámicas sociales de la Edad del Hierro no pueden ser entendibles a través de su comparativa con mecánicas y comportamientos observados a día de hoy, sería posible plantear la estrategia de concebir los conjuntos cerámicos de la Edad del Hierro como recipientes utilitarios con un sentido social propio. Al formar parte de un todo cultural, recogen y personifican las ideas de los esquemas sociales a los que pertenecen (Dobres, 2000). Ciertamente, una asunción muy positiva si nuestro objetivo radica en descubrir la importancia y función social del artesanado alfarero en la Edad del Hierro, aunque exija cambios en el ángulo tradicional de enfoque. 
Con carácter independiente a los estudios morformétricos que aportan informaciones útiles, los restos cerámicos también merecen ser investigados desde un punto de vista tecnológico. Sin embargo, no será hasta los años 90 cuando comiencen a surgir las primeras investigaciones que pongan de relieve el complejo panorama artesanal prerromano de la Península Ibérica (Escudero y Sanz Mínguez, 1993; García Heras, 1994; Blanco, 1998; Coll, 2000; Saiz Carrasco, 2005; Gutiérrez Cuenca et al., 2007; Burillo et al., 2008; Saiz y Gómez Villahermosa, 2008-2009; Sáez Romero, 2011; García Fernández y García Vargas, 2012; Gorgues y Benavente, 2012; Rodríguez González, 2012) (Fig. 3).

A tenor de las evidencias encontradas a día de hoy, podríamos plantear que, a partir del s. viII a. C. y en el sur peninsular, la adopción del torno iría cambiando progresivamente la manera de hacer cerámica (Vallejo, 2007), arrinconando poco a poco prácticas y técnicas anteriores caracterizadas por el modelado a mano. El yacimiento arqueológico de Las Cogotas (Cardeñosa, Ávila), aunque datado en un momento cronológico posterior, ss. III-II a. C., ofrece la posibilidad directa de rastrear la aparente conexión entre la introducción de cadenas tecnológicas basadas en el modelado a torno y la reafirmación de grupos patriarcales. Por un lado, el descubrimiento de todo tipo de herramientas y un número considerable de pellas - porciones- de barro en diversos contextos de habitación sugiere el desarrollo de una producción intradoméstica y vinculada al ámbito del hogar (Fig. 4). Una actividad que por otro lado convive, pero no compite, con la elaboración de cerámica a torno en un taller

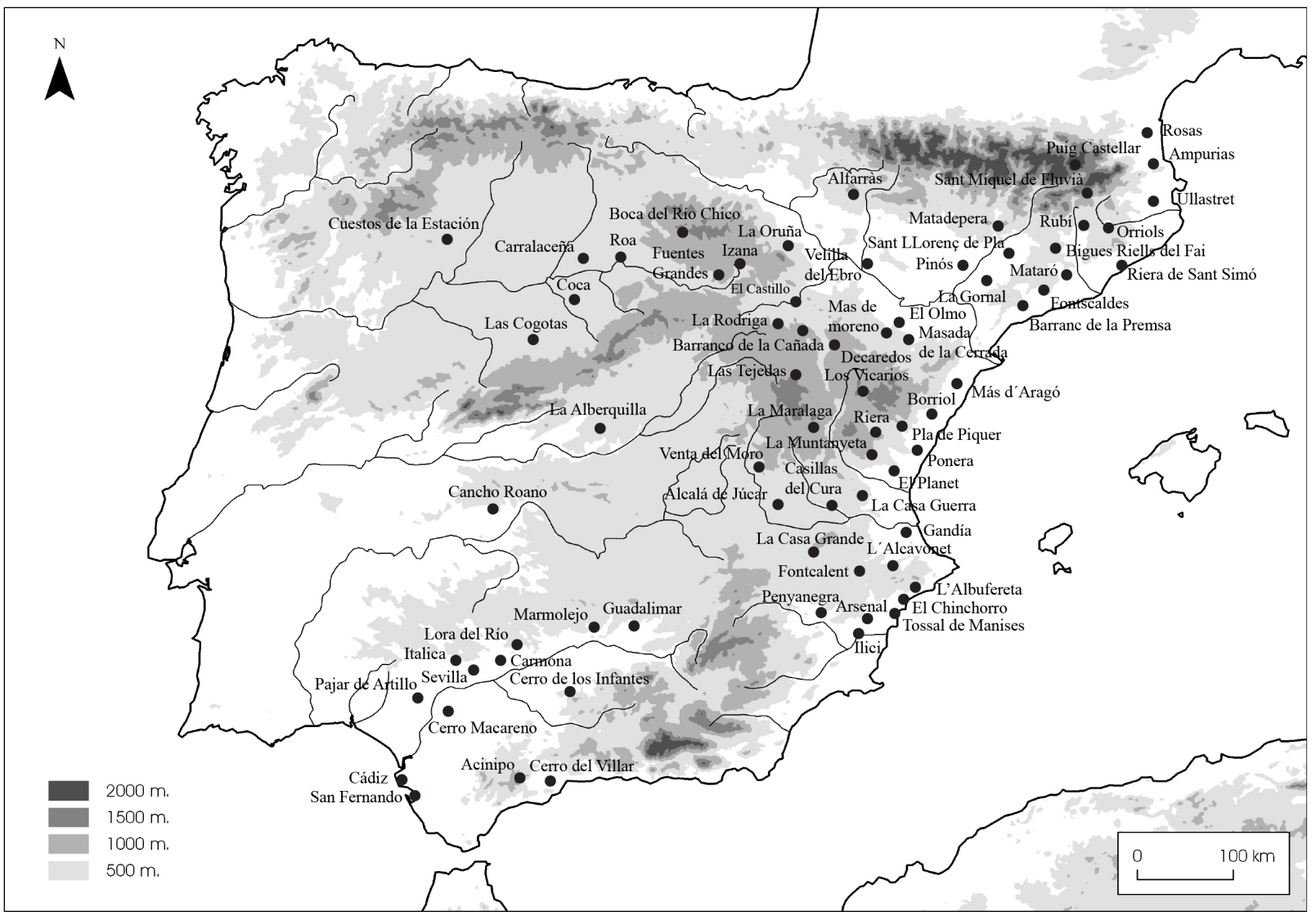

Fig. 3. Localización en la Península Ibérica de los yacimientos arqueológicos de época prerromana vinculados con el proceso productivo cerámico (a partir de Coll Conesa, 2000: 193, fig. 1). 


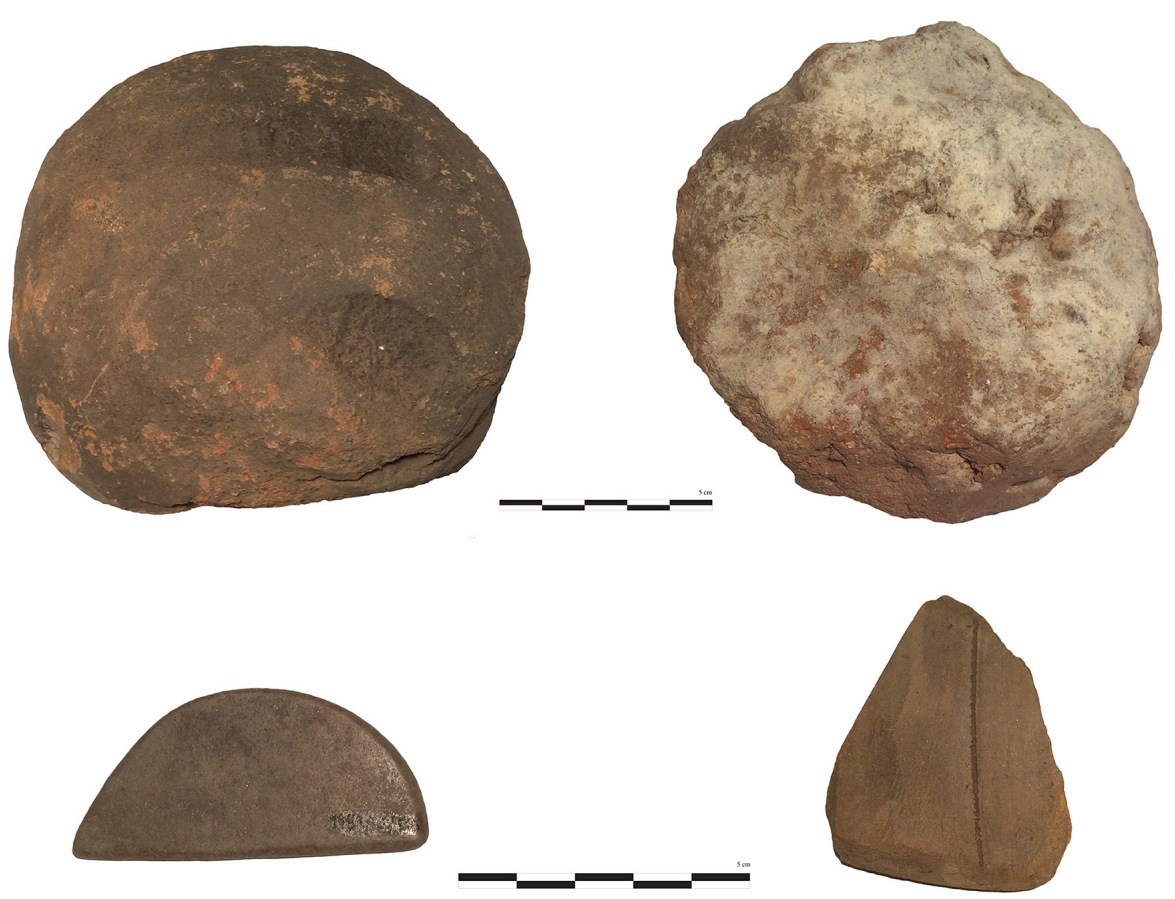

Fig. 4. Pellas de barro $\left(n .^{\circ} 35520 ; 1989-41-973\right.$ MAN) y herramientas para bruñir (canto rodado $=n .^{\circ} 1989-41-976$ MAN; galbo torneado $=n .^{\circ}$ 1989-41-977 MAN) halladas en el yacimiento arqueológico de Las Cogotas-Cardeñosa, Ávila-dentro de contextos de carácter doméstico.

ubicado en una zona a extramuros del Oppidum. Éste dispone de dos partes diferentes y perfectamente definidas, pero relacionadas entre sí (Fig. 5). En primer lugar, contamos con un área destinada esencialmente al trabajo y que, sin duda, fue testigo directo de la aplicación práctica de todos los gestos técnicos y las relaciones sociales derivadas de éstos. El registro de piletas de decantación cuadrangulares, canalizaciones hidráulicas, el anclaje de un pie de torno, espacios de secado y centenares de fragmentos con signos defectuosos de cocción evidencian la implantación de un procesado mecánico del mineral de arcilla, desde su obtención en el medio natural hasta la consecución del producto final. Su ejecución trajo consigo la materialización de terracotas no vitrificadas, de cochura oxidante y desgrasantes finos, con bordes exvasados y fondos en umbo, perfiles en " $s$ " o carenados, y decoradas con baquetones o motivos geométricos pintados (García Heras, 2005: 359).

El análisis macroscópico de sus pastas denota la intervención de agentes con un elevado grado de pericia y conocimientos tecnológicos. La preparación y decantación de las arcillas escogidas es meticulosa, no se aprecian fallos técnicos importantes relacionados con el levantado de la pieza, las aplicaciones de los correspondientes tratamientos de superficie suelen ser impecables, no suelen distinguirse roturas fruto de secados incontrolados y las coloraciones de cochura demuestran controles casi perfectos de la oxidación. Así mismo, en este ámbito han sido constatados ciertos materiales que hablan de la presencia de principiantes que intentaban emular las acciones efectuadas por los expertos. Además de distinguir muñecos y cajitas de barro no cocidas, fueron modeladas formas asimétricas y de pequeñas dimensiones, de paredes gruesas y repletas de irregularidades técnicas, hechas probablemente por individuos infantiles (Padilla y Chapon, 2015).

En segundo lugar, queda definido dentro del taller especializado un ámbito vinculado con lo estrictamente doméstico. Las campañas arqueológicas de los años 80 (Mariné y Ruiz Zapatero, 1988) pusieron al descubierto hogares y varias estancias, todas ellas repletas de conjuntos cerámicos, similares a los documentados por J. Cabré (1930: 76-78) en las casas excavadas intramuros, y otros elementos relacionados con la vivienda: afiladores, molinos, manos de molino, entre otros.

El alfar de Las Cogotas, el único complejo artesano de la protohistoria peninsular hasta ahora descubierto que conserva la totalidad de su superficie, no fue concebido para ser exclusivamente un espacio de trabajo. En él, producción y vida estaban 

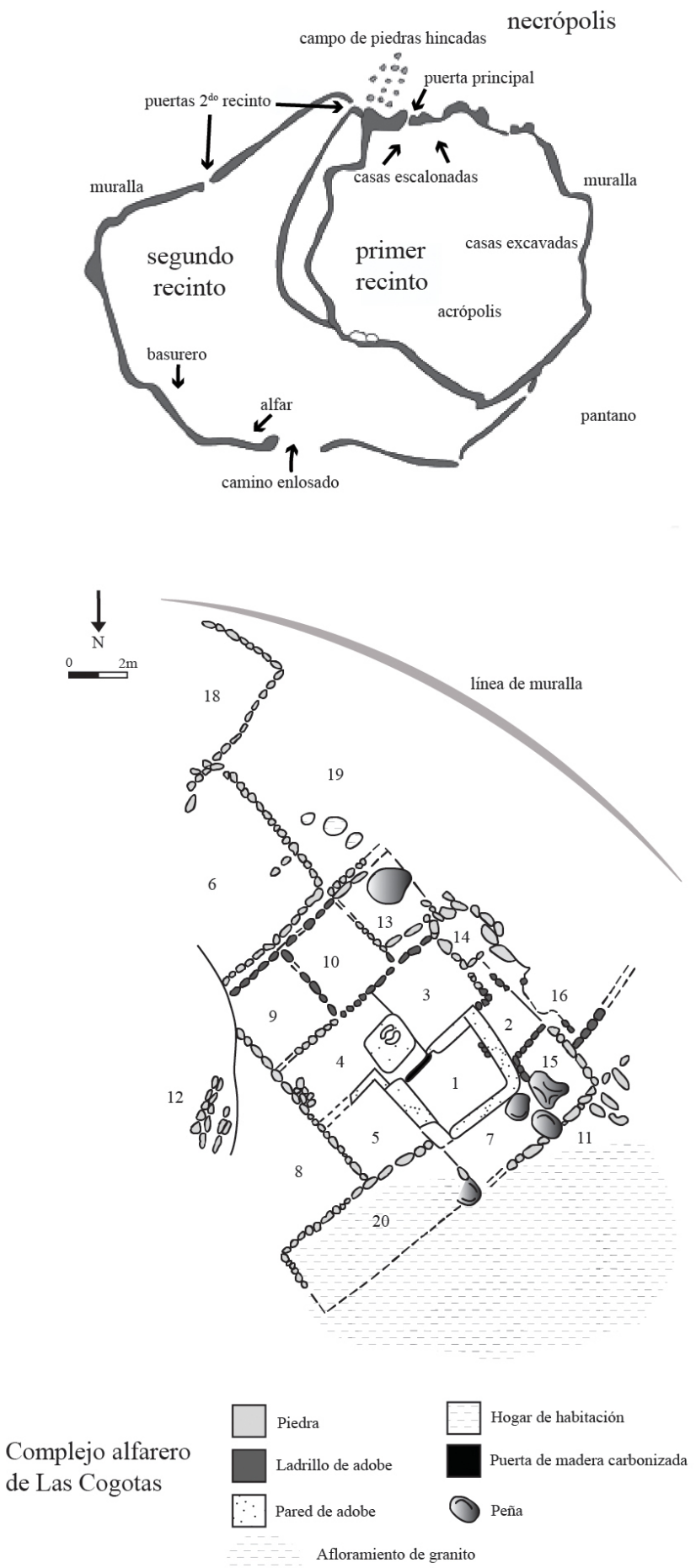

FIG. 5. Yacimiento arqueológico de Las Cogotas, Cardeñosa (Ávila): arriba, plano del sitio y abajo: planimetría detallada del complejo alfarero. íntimamente conectadas. La necesidad de apuntalar la emergencia de comunidades fuertemente jerarquizadas pudo promover el surgimiento de recintos específicos y destinados a la manufactura cerámica como éste, aparentemente ligados a una órbita familiar o de parentesco, pero regidos piramidalmente por un artesanado que fijaría su autoridad a partir de la habilidad y la experiencia práctica. Una hipótesis que, sorprendentemente, concuerda con lo que aparece plasmado en las famosas placas votivas de Penteskouphia (Corinto) o en la hidria atribuida al grupo de Leagros. En ellas, son representadas escenas protagonizadas por una serie de actores masculinos que escenifican fases concretas del trabajo cerámico, permitiendo intuir la presunta organización de un taller de tales menesteres en la Grecia del s. vi a. C. Teniendo en cuenta la subjetividad que a menudo ofrece lo iconográfico, en este caso y de forma curiosa, se reflejan cambios bien acentuados en el físico y vestimenta de los personajes, sugiriendo una diferenciación entre maestros alfareros y aprendices (Hasaki, 2012). También resulta interesante ver como las tareas de modelado y cocción suelen recaer en personas con barba, ataviadas con ropajes y bastones, mientras que otras etapas, tales como la decantación, son desarrolladas por sujetos en su mayoría imberbes y prácticamente semidesnudos (Díaz Rodríguez, 2014: 456).

¿Cómo debemos imaginar entonces el oficio alfarero durante la Edad del Hierro? Evidentemente, nos encontramos ante un interrogante bastante complejo y de difícil resolución, cuya respuesta parece estar vinculada a la estratificación y la consolidación de posiciones de "Poder" (Hernando Gonzalo, 2005, 2007). En definitiva, el establecimiento de un control tecnológico notorio que legitimaría, de forma directa o no, la imposición de una nueva realidad asentada en la individualidad de lo masculino. 


\section{Tras el impacto de Roma: dependencia y producción en masa}

Una de las principales carencias de la historiografía clásica ha sido la de concebir los objetos sin contexto y su interpretación en base a pensamientos positivistas y actualistas (David y Kramer, 2001; González Ruibal, 2012). La puesta en práctica de investigaciones orientadas en torno a estas premisas ha generado la visión del artesanado romano como una actividad de calidad y "alto nivel" (Rodríguez Neila, 2014: 39), de enorme importancia para el Imperio. Sin embargo, la materialización de nuevos estudios orientados hacia una perspectiva tecnológica pone de manifiesto la necesidad de replantear el paradigma establecido (Peña, 2007; Bermejo, $2011)^{2}$. Una vuelta de tuerca que sólo conseguiremos si, además de prestar interés en lo que la sociedad hace, nos centramos en cómo y por qué lo hace.

¿Qué ocurre cuando llega Roma? Tecnológica y técnicamente hablando, la estrategia productiva vuelve a sufrir cambios, que afectan de manera secuencial a cada una de las fases que intervienen en el proceso de manufactura cerámica, desde la obtención de la materia prima hasta la consecución del producto final (Fig. 6).

$\mathrm{Al}$ margen del factor humano, la producción cerámica requiere la conjunción de tres recursos fundamentales: agua, madera -combustible- y arcilla. Datos arqueológicos documentados a lo largo de la geografía peninsular constatan casi un millar de talleres alfareros, en su mayoría de medianas y grandes dimensiones, y articulados en torno a una serie de "focos de producción" (Díaz Rodríguez, 2014: 422-423) (Fig. 7). Todos ellos, ubicados intencionadamente en zonas conectadas con vías de comunicación (Juan Tovar, 1990; Ruiz Montes, 2014: 74-75) y próximas a fuentes hídricas y terrenos ricos en masa

2 Cf. también Marques da Silva, A. J.: "Does the ethnoarchaeology have a future beyond the analogy?". En Padilla, J. J. y Alarcón, E. (eds.): Etnoarqueología y Experimentación más allá de la analogía. Menga, en prensa.
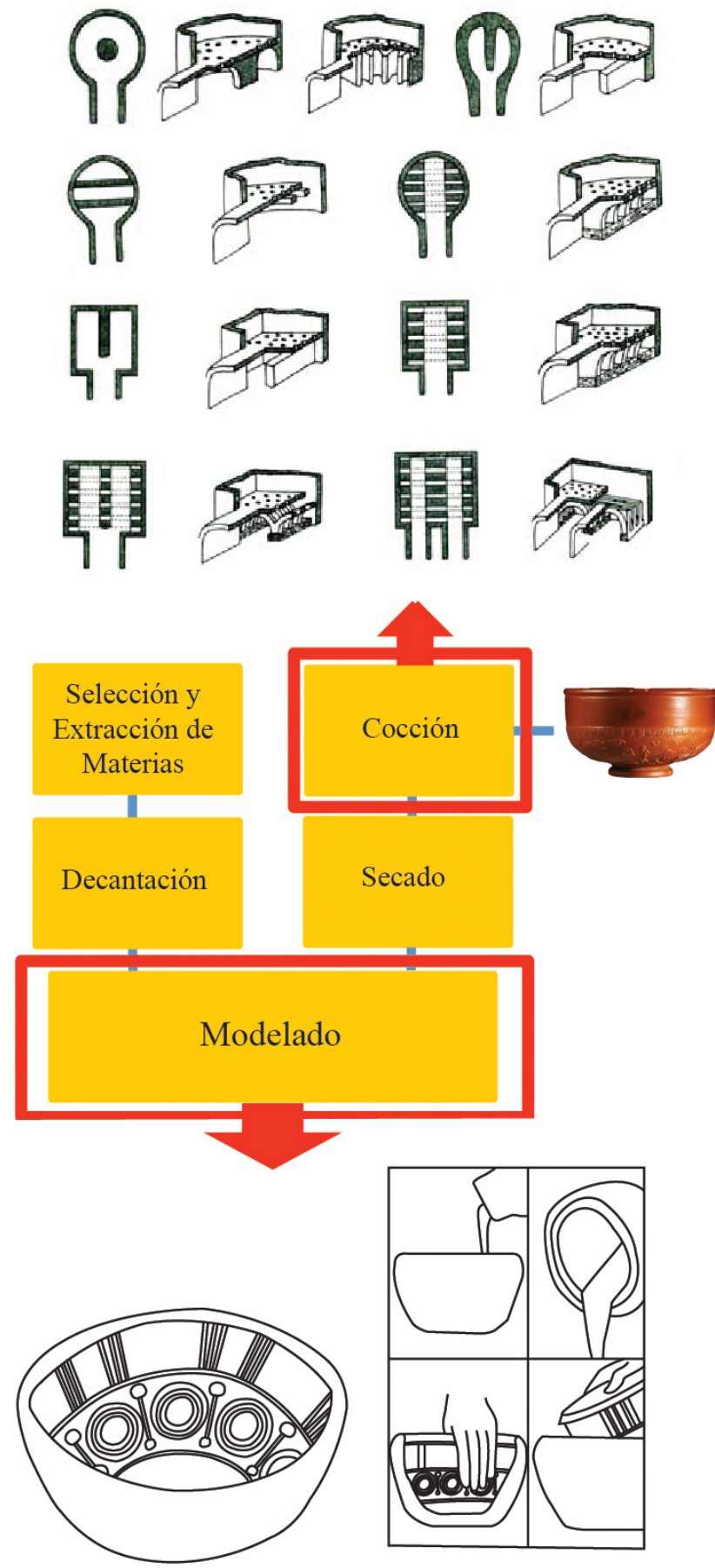

Fig. 6. Fases del proceso productivo cerámico en época romana; la introducción del modelado torneado a molde y el diseño de una tipología variada y funcional de estructuras de combustión se configuran como los cambios más importantes respecto al periodo prerromano (a partir de Cuomo di Caprio, 2007: 525). 


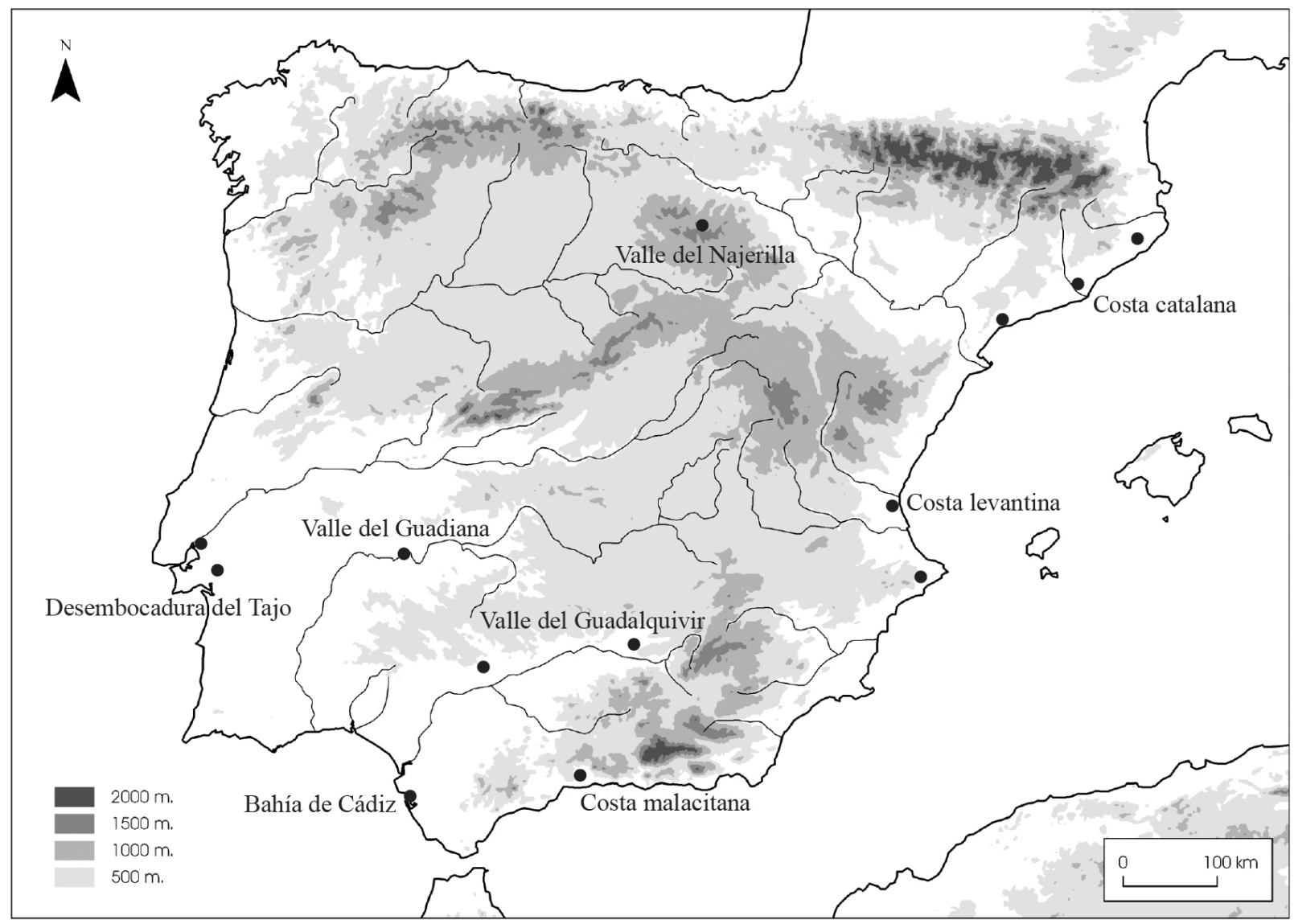

Fig. 7. Localización en Hispania de los principales focos de producción cerámica en época altoimperial (a partir de Díaz Rodriguez, 2014: 423, fig. 1).

forestal y arcillas. Así mismo, el hallazgo elevado y frecuente de restos cerámicos que aluden a tipos funcionales bien marcados, de cocina o común ${ }^{3}$, de almacenamiento y transporte (Buxeda i Garrigós et al., 2006) o vajilla fina o de mesa ${ }^{4}$ (Sáenz Preciado, 1999), respalda la hipótesis del inicio de una explotación intensiva del territorio, fundamentalmente para el acopio de grandes y heterogéneas cantidades de mineral. Sería establecido, por tanto, un aprovechamiento masivo de toda clase de filones

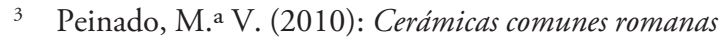
en el Alto Guadalquivir. Tesis doctoral inédita presentada en 2010 en la Univ. de Granada.

4 Ruiz Montes, P. (2012): Nuevas lecturas en torno a procesos de producción y sustratos productivos en el complejo alfarero romano de Los Villares de Andújar (Andújar, Jaén). Tesis doctoral inédita presentada en 2012 en la Univ. de Granada. o cuencas sedimentarias, siendo éstas, en ocasiones, no necesariamente óptimas por su calidad y grado de impurezas. Un comportamiento que explicaría perfectamente la presencia de elevadas cantidades de carbonato cálcico, cuarzo y mica en la matriz de determinados fragmentos (Fig. 8).

Otro factor que podría aclarar el porqué de la numerosa presencia de estos carbonatos primarios e impurezas en las pastas de época romana guarda relación con la decantación del sólido arcilloso. La necesidad de depurar una suma ingente de materia prima pudo incidir en el desarrollo de un grado de precisión menor en el descarte de estos elementos durante el proceso de levigado. Además, el reconocimiento visual en las matrices de grandes proporciones de clastos redondeados y sin aristas angulosas descartaría un posible machacado intencional para su adición. La no eliminación de estas impurezas 
a través de piletas, repletas de agua y comunicadas entre sí, conlleva generalmente fallos postcocción a partir de los $800^{\circ} \mathrm{C}$, ocasionando ciertas alteraciones internas y defectos externos en el producto final (Belde y Druc, 1999; Cultrone et al., 2001; Gámiz Caro et al., 2013; Quinn, 2013), siendo éstos bastante comunes en los restos cerámicos que se conservan. Concretamente nos referimos a la constatación de cuerpos arcillosos poco consistentes y barnices agujerados y agrietados. También, en una buena parte de los tipos cerámicos fabricados según los cánones de Roma es posible distinguir fracturas y craquelados motivados, frecuentemente, por burbujas de aire que permanecen en su interior (Sempere, 1992: 191-192). Con toda seguridad, éstas vendrían condicionadas por una ejecución no correcta del pisado y amasado, dado el grueso de material que debía ser homogeneizado.

El nacimiento del concepto de “tipo" y la producción de un elevado volumen de elementos cerámicos fuertemente estandarizados (Juan Tovar, 1990; Ruiz Montes, 2011) marcan transformaciones más que evidentes en la fase de modelado. El horizonte arqueológico permite discernir el mantenimiento efectivo del torno como herramienta, pero un cambio radical a nivel técnico. La introducción del molde para la fabricación de un grupo concreto de bienes - terracotas, lucernas o vajillas de terra sigillata- generó una auténtica revolución productiva que multiplicó la cuota de elaboración de piezas y redujo la importancia de la habilidad y experiencia práctica como factores de control tecnológico (Fig. 6).

A tenor de las evidencias, el secado no experimentaría variaciones destacables, todo lo contrario a lo acaecido dentro de los formatos decorativos. Además

del empleo de moldes (Fernández y Serrano, 2013; Roca et al., 2014) para plasmar frisos iconográficos y ornamentales variados, usaron punzones, buriles y técnicas como la barbotina. Igualmente y tras su secado inmediato, tipos determinados eran bañados con engobes de arcilla, buscando en determinados 
casos la consecución de un prototipo de barniz. El contexto arqueológico nos muestra engobes y barnices de calidad variable (Ruiz Montes, 2014), que no han perdurado, o bien, presentan grietas o estrías que rompen la superficie añadida. Un hecho justificable, si tenemos en cuenta el apremio por fabricar elementos materiales en masa y, por ende, el escaso control en la preparación de las mezclas químicas, seguramente poco consistentes y exactas.

Junto al modelado, la fase de cocción presenta las modificaciones de mayor entidad. La información aportada por los yacimientos excavados (Sotomayor, 1970; Girón, 2011; Bustamante, 2013; Tremoleda y Castanyer, 2013) permite corroborar una cierta continuación tecnológica, aunque con alteraciones radicales en cuanto a la aplicación del gesto técnico. La proliferación de complejos alfareros de entidad considerable, probablemente en base a la intencionalidad de satisfacer las prerrogativas del imperio, motivó la aparición de un mayor número de estructuras de combustión y una creciente y variada tipología de éstas (Cuomo Di Caprio, 2007) (Fig. 6). A ciencia cierta, la morfología - planta circular o cuadrangular- y particularidades específicas de estos hornos - disposición de cubierta permanente o no, pilastras centrales, muros axiales, arcadas con corredor, etc.- estarían supeditadas a las propiedades prefijadas de los objetos dispuestos en su interior.

A pesar de contar con ejemplos que certifican la pervivencia de la tradición indígena (Abascal, 2008), la dominación de Roma configura otra manera de hacer cerámica. Cabe, pues, preguntarse ahora el porqué de este cambio. Una pregunta, sin duda, de difícil solución, que tan sólo seremos capaces de intentar resolver si logramos indagar en la verdadera "lógica social" de la materialidad que estudiamos 5 . Es decir, la estructuración de aquellas relaciones existentes entre los objetos y las dinámicas sociales que caracterizan el grupo humano al que se adscriben. El modo de elaborar cerámica no es ajeno

5 González Ruibal, A.: "La lógica social de la cerámica. Un ejemplo etnoarqueológico". En Padilla, J. J. y Alarcón, E. (eds.): Etnoarqueología y Experimentación más allá de la analogía. Menga, en prensa. a otras "prácticas o valores sociales" (Gosselain, 2011: 223), determinando en ocasiones permutas tecnológicas y variantes técnicas.

El aparato estatal romano instaura un régimen sustentado en estructuras sociales, económicas e ideológicas, que potencian aún más, si cabe, la masculinidad individualizada y el poder. El análisis de la Cadena Técnico-Operativa desde una perspectiva bourdiana nos acerca a un contexto social de mayor jerarquización basado en la riqueza y el consumo, que fija un dominio fuerte en todos los ámbitos de la cultura. El habitus técnico cerámico vuelve a cambiar, fortaleciendo de forma indirecta el nuevo orden impuesto (Fig. 9).

Se fomenta un sistema de masas, definido en ocasiones como "protoindustrial" (Dark, 2001), que otorga superioridad a pequeñas élites. A través del control de los recursos y medios de producción, se consolida un modelo de vida asentado de forma quasi exclusiva en lo funcional, lo meramente estético y lo competitivo. Por medio de la estandarización, el dinamismo económico altoimperial diluye la importancia que antańo tuvo el conocimiento como mecanismo de atribución. El artesanado cambia como concepto, quedando vinculado de forma exclusiva al término de productividad. La puesta en valor de lo meramente cuantitativo origina una cultura cerámica repetida, de mismas características y propiedades, diferenciadas únicamente por el uso para el que han sido preconcebidas. El afianzamiento de un comercio supra- e interregional (Sáenz Preciado, 2014: 149) demanda, a su vez, la elaboración de bienes idénticos, que sólo sirven para satisfacer necesidades puntuales. Se conforma, por tanto, un trabajo monótono y rutinario, definido por un mismo grado de pericia técnica. La habilidad comienza a ser regulada con el propósito de concebir productos estandarizados (Longacre, 1999), a costa, eso sí, de una pérdida de calidad y la formación de falsos especialistas.

La adscripción a nuevas modas y estilos de vida legitima, de manera consciente o no, la realidad social instalada tras la conquista. Utilizar recipientes cerámicos normalizados en determinados casos, incluso, con composiciones decorativas idénticas, 

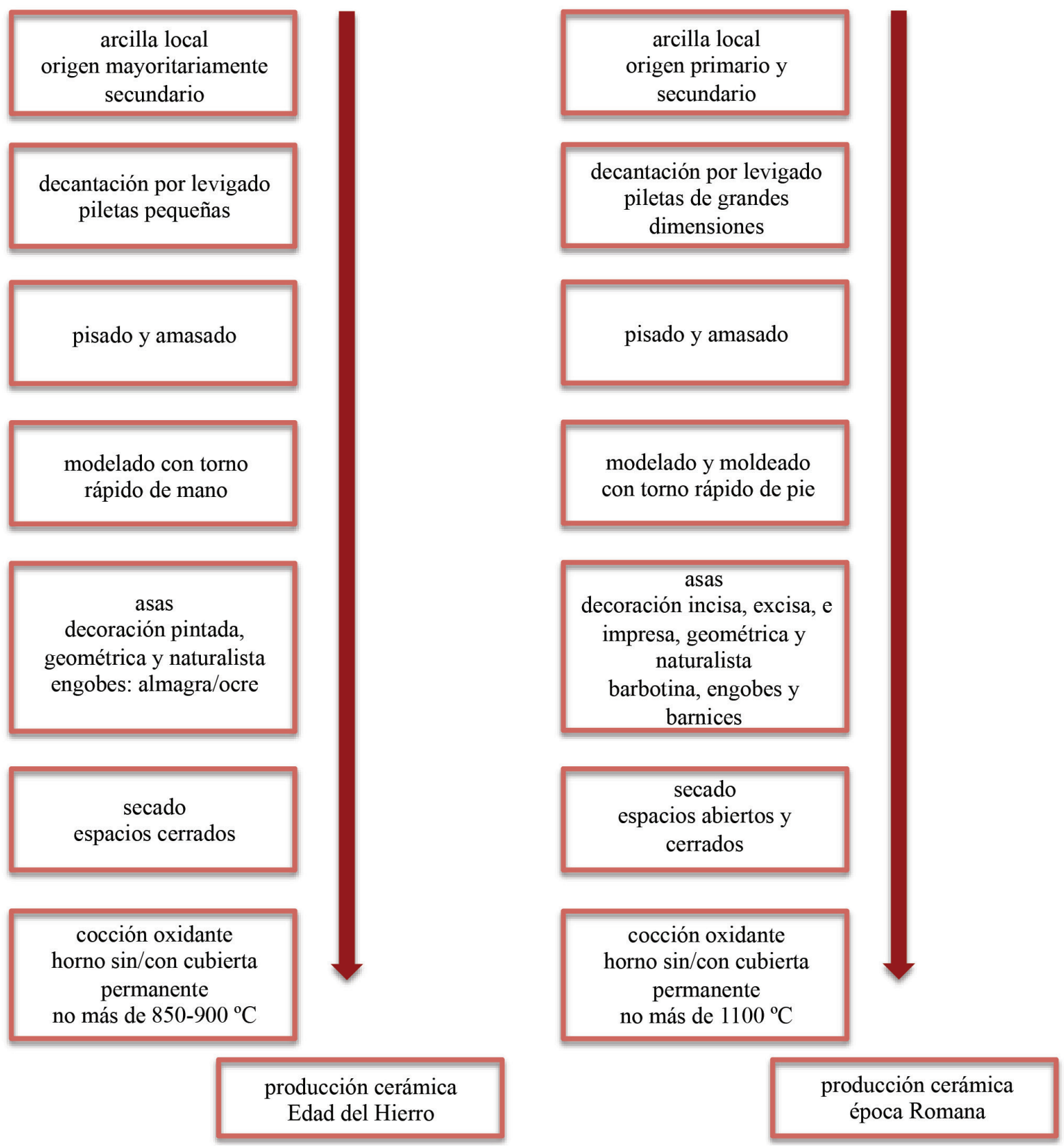

Fig. 9. Cadenas Técnico-Operativas documentadas para la producción cerámica en la Edad del Hierro y a comienzos de la dominación romana.

justifica el poder de Roma. Un poder cimentado en la explotación industrial y comercial de bienes y la supeditación de toda una población frente a unos pocos ediles públicos o privados. Los artesanos pasan a ser simples trabajadores que tan sólo crean productos para una entidad superior, siendo, por esto, marginados y desvalorados. Independientemente de su condición, libres, libertos o esclavos, se convierten en personas subordinadas, al servicio de grandes propietarios, que realizan en exclusiva lo que le es encargado. El savoir-faire técnico (García Rosselló y Calvo, 2013: 20) se mecaniza y queda 
reducido a tareas prefijadas, motivando una limitación de la maestría.

Autores clásicos, como Cicerón (De off., I, 42, 150) o Séneca (Epist. Mor. 88, 20), recogen en sus escritos el menosprecio de la comunidad hacia los que ofrecían sus servicios a cambio de una remuneración, precisamente por estar en una situación de dependencia. Independientemente de su veracidad real o no, estos relatos defienden, a la vez que respaldan, la reconstrucción cultural impuesta por los que detentan el poder. Conjuntamente, otros textos, firmados por Varrón (De Re rústica, 1, 2, 22-23) y Ulpiano (Dig., 50, 16, 198), abordan con todo lujo de detalles la organización efectiva de la producción y la propiedad de las arcillas, haciendo especial hincapié en las relaciones de supeditación del officinator y el dominus, propietario de los praedia-figlinae (Juan Tovar, 1990: 31). Fueron promulgados, además, una serie de pasajes jurídicos, como los contenidos en la lex Ursonensis (Tsiolis, 1997; Cinca, 2000; Fernández Baquero, 2016), que refrendan la necesidad de naturalizar el intervencionismo y el dominio de una pequeña élite, aludiendo mayoritariamente, en este caso, a la fabricación y consumo de materiales cerámicos de construcción.

El deseo de desvinculación del grupo llega a su cenit, constituyéndose una individualidad masculina que discrimina la masculinidad en sí misma. Se produce, en definitiva, una redefinición del patriarcado que, al margen de reprimir lo puramente emocional y femenino, silencia también el papel individualista desempeñado por ciertos grupos de hombres. De hecho, la organización de asociaciones profesionales colectivas como los collegia, creados supuestamente para velar por los intereses de quienes ejercían una misma ocupación artesana, no haría sino ratificar en primera persona cada uno de los principios que sustentarían su conveniente desigualdad.

Sin embargo, y como ya apuntábamos brevemente un poco más arriba, en ocasiones, la materialidad cerámica típica de contextos imperiales revela muestras de continuidad. Nos referimos a la designada "cerámica pintada romana de tradición indígena" o "tipo Clunia", de la que tan sólo existen investigaciones centradas en sus características formales y, por ende, finales (Luezas y Martín Bueno, 1995; Abascal, 2008). El descuadre con el sobreentendido progreso cultural de lo romano, afín al alegato recurrente de la modernidad (Hernando, 2012), ha motivado la marginación historiográfica de estos restos y sus respectivas secuencias, obviando por completo una parte importante del verdadero discurso histórico acaecido. Entendidas como "novedades significativas", las modificaciones tipológicas centran el discurso explicativo, sin ahondar en sus propiedades técnicas. Queda asumida la conversión del proceso productivo, necesaria para alcanzar el nivel de las plenamente romanas y estar en condiciones de competir comercialmente con ellas. No obstante, si analizamos con detenimiento la tecnología de estos vasos nos percatamos de todo lo contrario, una pervivencia manifiesta de la huella de calidad indígena (Blanco, 2012) (Fig. 10).

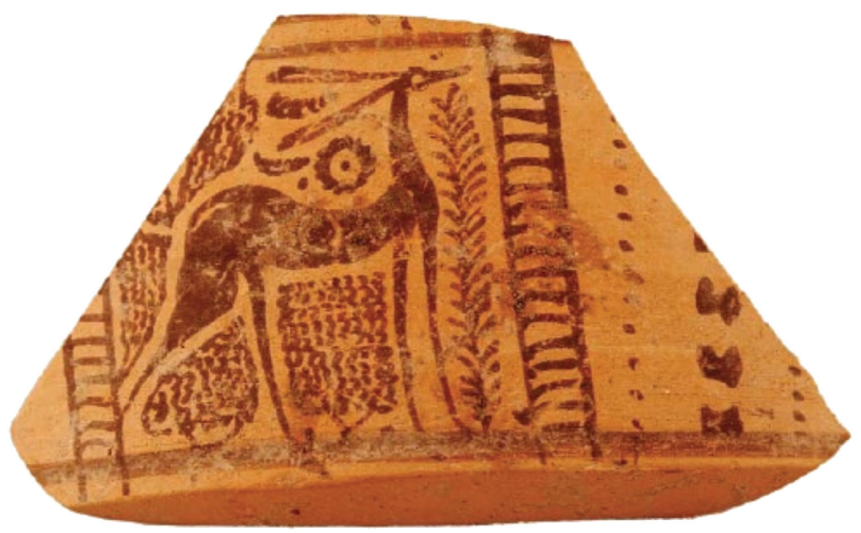

Fig. 10. Fragmento cerámico de 'tipo Clunia' hallado en el yacimiento de Pintia, Padilla de Duero (Valladolid) (según Blanco García, 2012: 56).

Apareciendo alternativamente hasta el s. II d. C., este grupo cerámico evidenciaría, quizás, un sentimiento de resistencia al modelo impuesto. Evidentemente, las fuentes literarias del momento olvidan por completo esta modalidad de producción, haciendo hincapié en el funcionamiento de un engranaje social y económico perfecto, que rige con entereza un régimen de vida acaparado por la uniformidad y homogeneidad. La certeza del consumo 
de cerámica pintada en época altoimperial demostraría un panorama social distinto y repleto de fisuras, en el que talleres artesanos seguirían perpetuando el habitus cerámico heredado de generaciones anteriores y elaborando piezas para comunidades no tan susceptibles al impacto de la conquista de Roma.

Retomar la senda de tales producciones teniendo en cuenta otros parámetros ayudaría a comprender el porqué del deseo de hacer perdurar determinadas costumbres y fórmulas de subsistencia, así como el firme rechazo de leyes más férreas (Scott, 2000), que ponían el énfasis en una mayor explotación económica y consecuentemente en una creciente desigualdad. Próximos trabajos deberían, sin duda, emprender la búsqueda de respuestas concretas.

\section{Conclusiones}

Estas líneas recalcan la importancia de analizar la cultura material a partir de lecturas alternativas y diferentes a las preconizadas tradicionalmente. Es decir, tratar de entender la cerámica no como un producto final con unas características formales y funcionales claras, sino como un continente lleno de valor que encierra en su interior todo tipo de códigos de significado.

Llevar a cabo estudios que aborden en profundidad el proceso productivo de los conjuntos cerámicos peninsulares a finales de la Edad del Hierro permite indagar también en el universo de aquellas comunidades encargadas de su manufactura y consumo; y, por supuesto, percibir de igual manera la transformación paulatina, sobre todo de las esferas de lo social y lo económico, tras la llegada de Roma.

La incorporación del torno como herramienta para la fabricación de elementos cerámicos y la organización de la producción a través de esta nueva tecnología consolidaron la afirmación de grupos muy jerarquizados y garantes de una identidad cada vez más individualizada. La idea del "yo" masculino frente a lo colectivo y femenino (Ruiz Gálvez, 2013: 223) sería reforzada mediante el control del conocimiento, surgiendo un nivel de organización social estructurado en base a una autoridad fijada por la habilidad y la experiencia práctica. La producción de piezas torneadas, caracterizadas principalmente por su alto nivel de calidad, otorgarían estatus y poder, al mismo tiempo que fortalecerían el orden patriarcal.

En cambio, el dominio de Roma trajo consigo nuevas dinámicas que romperían progresivamente con tradiciones anteriores. La búsqueda exacerbada de la individualidad conllevaría la articulación de un sistema reglamentado por una entidad estatal y dirigido por una pequeña élite masculina, que ejercería su potestad a través del control de las necesidades de un Imperio. El artesanado cerámico se convertiría así en una pieza más del motor que pondría en marcha el desarrollo de otro patrón de vida. La Cadena Técnico-Operativa sufrió cambios efectivos que afectarían de igual modo a los agentes sociales intervinientes en su materialización. El establecimiento de una producción en masa generó piezas estandarizadas, con una escasa variedad formal y atributos plenamente funcionales. La cantidad prevalecería sobre la calidad, un hecho que motivaría la conversión de los alfareros en falsos especialistas al servicio de identidades individualizadas en su máxima expresión (Hernando, 2002). No obstante, la constatación en ciertos casos del mantenimiento de técnicas y ejemplares híbridos, encuadrados dentro de la categoría genérica de cerámicas romanas de tradición indígena, parecen oponerse a dicha institucionalización, narrando por sí mismos un relato bastante más complejo de lo asumido habitualmente. Desgraciadamente, pocos son los estudios que ahondan en estas producciones (Taracena, 193132; Abascal, 1986 y 2008; Ruiz Valderas, 1988; Luezas y Martín Bueno, 1995; Sánchez Simón, 1995; Polo, 1999; Bustamante, 2016; Río-Miranda, 2017), convirtiéndose en un reto atrayente y sugerente de cara a investigaciones futuras. Quizás, el análisis tecnológico de estos recipientes permita por fin poner cara a los alfareros de aquellas poblaciones que, a pesar de la instauración de nuevas formas de vivir, querrían seguir almacenando, comiendo y bebiendo en cerámicas anaranjadas decoradas con pinturas rojas y negras. 
En definitiva, se pretende dar comienzo a una reflexión profunda que cuestione asunciones históricas forjadas en visiones actualistas y que, a menudo, no reflejan el carácter efectivo de lo acontecido. En nuestro caso, debemos romper con la percepción que tenemos del término "progreso", siendo éste en ocasiones un signo que no siempre es sinónimo de "evolución".

\section{Bibliografía}

Abascal, J. M. (1986): La cerámica pintada romana de tradición indigena en la Península Ibérica. Madrid: Univ. de Alicante.

Abascal, J. M. (2008): "Las cerámicas 'tipo Clunia' y otras producciones pintadas hispanorromanas". En Bernal, D. y Ribera, A. (eds.): Cerámicas hispanorromanas. Un estado de la cuestión. Cádiz: UCA, pp. 429-443.

Balfet, H. (1975): “Technologie”. En Creswell, R. (ed.): Éléments d'ethnologie, vol. 2. Paris: Armand Colin, pp. 44-79.

Bermejo, J. (2010): "Poder, ideología y espacio: la teoría de la producción espacial. Algunas aplicaciones arqueológicas". En Navarro, G. M.; Estévez, B. y SÁnchez Cuervo, A. (coords.): Claves actuales de pensamiento. Madrid: csic, pp. 435-454.

Bermejo, J. (2011): "La cerámica común de mesa, cocina y despensa en el alto Duero durante el periodo bajoimperial: Un ensayo de clasificación”, Espacio Tiempo y Forma. Serie I. Nueva época. Prehistoria y Arqueología, 4, pp. 337-358.

Bermúdez, A. y Juan Tovar, L. C. (1990): "Las fuentes clásicas en el estudio de las industrias cerámicas", Anuario de la Univ. Internac. SEK, 1, pp. 23-35.

Bernal, D. y Ribera, A. (2008): Cerámicas hispanorromanas. Un estado de la cuestión. Cádiz: UCA.

Bernal, D. y Ribera, A. (2012): Cerámicas hispanorromanas II. Producciones regionales. Cádiz: UCA.

Blanco, J. F. (1998): "Las producciones cerámicas del alfar de Cauca (Coca, Segovia)", Madrider Mitteilungen, 39, pp. 121-141.

Blanco, J. F. (2012): "Animales salvajes en el imaginario vacceo", Vaccea, 5, pp. 52-59.

Bourdieu, P. (1988 [1979]): La distinción. Criterio y bases sociales del gusto. Madrid: Taurus.

Bowser, B. J. (2000): "From pottery to politics: An Ethnoarchaeological study of political factionalism, ethnicity, and domestic pottery style in the Ecuadorian Amazon", Journal of Archaeological Method and Theory, 7 (3), pp. 219-248.

Burillo, F.; Cano, M. ${ }^{a}$ A. y Sáiz Carrasco, M. ${ }^{a}$ E. (2008): "La cerámica celtibérica". En Bernal, D. y Ribera, A. (eds.): Cerámicas Hispanorromanas. Un estado de la cuestión. Cádiz: UCA, pp. 171-188.

Bustamante, M. (2013): "El trabajo artesanal en Augusta Emerita durante los ss. I-IV d. C.", Zephyrus, LXXII, pp. 113-138.

Bustamante, M. (2016): "La cerámica pintada romana de tradición indígena en el territorio de Extremadura”, SPAL, 25, pp. 183-207.

Buxeda, J.; Martínez Ferreras, V. y Vila, L. (2006): "Les primeres produccions d'àmfores romanes a la Tarraconense. Per una arqueometria del canvi tecnològic, de la producción i del consum". En CARRERAS, T. (ed.): La producción i el comerc de les àmfores de la Provincia Hispania Tarraconensis. Monografies, 8. Barcelona: MAC, pp. 151-161.

Cabré, J. (1930): Excavaciones en Las Cogotas, Cardeñosa (Ávila) I. El castro. Junta Superior de Excavaciones y Antigüedades, 110. Madrid.

Calvo Trías, M. y García Rosselló, J. (2012): “Tradición técnica y contactos: Un marco de reflexión centrado en la producción cerámica", Rubricatum, 5 , pp. 393-401.

Cárdenas, A. y Agudo, J. A. (2012): La Edad del Barro. Granada: Port-Royal.

Childe, V. G. (1954): "Rotary motion". En Singer, C.; Holmyard, E. J.; Hall, A. R. y Williams, T. I. (eds.): A History of Technology. London: oup, pp. $187-215$.

CincA, J. L. (2000): "Elementos de alfar en el casco urbano de Calahorra. ¿Un nuevo alfar de producción de cerámica romana?”, Iberia, 3, pp. 319-332.

Coll, J. (2000): “Aspectos de tecnología de producción de la cerámica Ibérica”. En Mata, C. y Pérez Jordá, G. (eds.): III Reunió sobre Economía en el Món Iberic. Saguntum Extra, 3. Valencia, pp. 191-208.

Cooper, E. (2000): 10.000 years of Pottery. London: BMP.

Cresswell, R. (1976): “Avant-Propos”, Techniques et Culture, 1, pp. 5-6.

Cultrone, G.; Rodríguez Navarro, C.; Sebastián, E.; Cazalla, O. y De La Torre, M. J. (2001): "Carbonate and silicate phase reactions during ceramic firing", European Journal Mineral, 13, pp. 621-634.

Cuomo Di Caprio, N. (2007): Cerámica in Archeologia 2: antiche tecniche di lavorazione e moderni metodi di 
indagine. Studia Archaeologica, 144. Roma: L'Erma di Bretschneider.

DARK, K. (2001): "Proto-industrialization and the Economy of the Roman Empire". En Polfer, M. (ed.): L'Artisanat romain : évolutions, continuités et ruptures (Italie et provinces occidentales). Actes 2ème colloque d'Erpeldange, 2001. Monographies Instrumentum, 20. Montagnac: Éditions Monique Mergoil, pp. 19-29.

David, N. y Kramer, C. (2001): Ethnoarchaeology in action. Cambridge/New York: cup.

Díaz Rodríguez, J. J. (2014): “Algo más que hornos y cerámicas. La manufactura alfarera en la antigüedad altoimperial hispanorromana: entre el artesanado y la producción en masa”. En Bustamante, M. y Bernal, D. (eds.): Artifices idóneos. Artesanos, talleres $y$ manufacturas en Hispania. Mérida-Madrid: CsIC, pp. 13-42.

Dietler, M. y Herbich, I. (1998): "Habitus, techniques, style: an integrated approach to the social understanding of material culture and boundaries". En StARK, M. (ed.): The Archaeology of Social Boundaries. Washington: Smithsonian, pp. 232-279.

Djorjevic, B. (2012): Intangible cultural heritage. An analysis of the traditional pottery production related legislation. Belgrado: NMB.

Dobres, M. A. (2000): Technology and social agency. London: Blackwell.

Duistermaat, K. y Groot, N. (2008): "A new ethnoarchaeology documentation proyect at the Fustat Pottery workshops, Egypt", Leiden Journal of Pottery Studies, 24, pp. 181-186.

Escudero, Z. y Sanz Mínguez, C. (1993): “Un centro alfarero de época vaccea: el Horno 2 de Carralaceña (Pesquera de Duero, Valladolid)". En Romero, F.; Sanz Mínguez, C. y Escudero, Z. (eds.): Vaccea. Estudios sobre el mundo prerromano en la cuenca media del Duero. Valladolid: Jcyl, pp. 471- 492.

Fernández Baquero, M. E. (2016): "Límites a la construcción de alfarerías en la Lex Ursonensis", Anuario Jurídico y Económico Escurialense, xLIX, pp. 63-88.

Fernández García, M. I. y Serrano, B. (2013): "Estructuras humanas de producción: fabricantes de moldes en Los Villares de Andújar (Jaén, España)". En Bernal Casasola, D.; Juan Tovar, L. C.; Bustamante, M.; Díaz Rodríguez, J. J. y Sáez, A. M. (eds.): Hornos, talleres y focos de producción alfarera en Hispania. Monografías Ex Officina Hispana, 1. Cádiz, pp. 251-256.

Foucault, M. (1992 [1975]): Vigilar y Castigar. Nacimiento de la prisión. Madrid: Siglo xxI.
Gámiz, J.; Dorado, A. y Cabadas, H. V. (2013): “Análisis de cerámica prehistórica con estereomicroscopía: una guía revisada sobre la descripción de las fases de producción", Cuadernos de Prehistoria y Arqueología de la Univ. de Granada, 23, pp. 365-385.

Gandom, E.; Bootsma, R. J.; John, A.; Endler, J. A. y Leore Grosman, L. (2013): "How Can Ten Fingers Shape a Pot? Evidence for Equivalent Function in Culturally Distinct Motor Skills", PLOS one, 8, pp. 1-9.

García Fernández, F. J. y García Vargas, E. (2012): "Los hornos alfareros de tradición fenicia en el valle del Guadalquivir y su perduración en época romana: Aspectos tecnológicos y sociales", SPAL, 21, pp. 9-38.

García Heras, M. (1994): "El yacimiento celtibérico de Izana (Soria). Un modelo de producción cerámica”, Zephyrus, XLVII, pp. 133-155.

García Heras, M. (2005): "La tecnología cerámica”. En Jimeno, A.; De la Torre, J. I. y Chaín, A. (coords.): Celtíberos: Tras la estela de Numancia. Soria: Diput. de Soria, pp. 359-366.

García Roselló, J. (2008): Etnoarqueología de la producción cerámica. Identidad y territorio en los Valles Centrales de Chile. Mayurqa, 32. Palma de Mallorca.

García Rosselló, J. y Calvo Trías, M. (2013): Making Pots. El modelado de la cerámica a mano y su potencial interpretativo. BAR Intern. Ser., 2540. Oxford: Archaeopress.

Giddens, A. (2003): La constitución de la sociedad. Bases para la teoría de la estructuración. Buenos Aires: Amorrortu Edit.

Girón, L. (2011): "Espacio físico y social: 'espacio arqueológico'. Estado de la cuestión y propuesta de estudio para la zona industrial de la Bahía de Cádiz en época romana”, Estrat Crític, 5 (1), pp. 254-263.

González Ruibal, A. (2005): "Etnoarqueología de la cerámica en el oeste de Etiopía”, Trabajos de Prehistoria, 62 (2), pp. 41-66.

GonzÁlez Ruibal, A. (2012): "Hacia otra arqueología: Diez propuestas", Complutum, 23 (2), pp. 103-116.

Gorgues, A. y Benavente, J. A. (2012): "Organisation du travail et technologie potière dans les ateliers ibériques tardifs du Mas de Moreno (FozCalanda, Teruel): Bilan provisoire des recherches (2005-2011)". En Belarte, M. C.; Benavente, J. A.; Fatás, L.; Diloli, J.; Moret, P. y Noguera, J. (coords.): Actas II Congreso Internacional Iberos del Ebro. Tarragona: ICAC, pp. 273-290.

Gosselain, O. P. (2002): Poteries du Cameroun Meridional. Styles, Techniques et raports à l'identité. Paris: CNRS. 
Gosselain, O. P. (2011): “Fine if I do, fine if I don't. Dynamics of technical knowledge in Sub-Saharan Africa”. En Roberts, B. W. y VAn Der Linden, M. (eds.): Investigating archaeological cultures. Nueva York: Springer, pp. 211-227.

Gutiérrez Cuenca, E.; Muñoz Fernández, E.; Morlote, J. M. y Montes Barquín, R. (2007): "El horno de La Alberquilla, un centro productor de cerámica carpetana en Toledo", Zona Arqueológica, 10 (2), pp. 303-323.

Gutiérrez Rodríguez, M.; Orfila Pons, M. y SánCHez López, E. (2017): "La identificación del catastro rural a través de los fundi. Una metodología aplicada en el ager iliberritanus", Zephyrus, LXxix, pp. 103-125.

Hasaki, E. (2012): "Craft Apprenticeship in Ancient Greece. Reaching beyond the Masters". En WeNDRICH, W. (ed.): Archaeology and apprenticeship. Body knowledge, identity, and communities of practice. Arizona: UAP, pp. 171-202.

Hernando, A. (2002): Arqueología de la identidad. Madrid: Akal.

Hernando, A. (2005): "Mujeres y Prehistoria. En torno a la cuestión del origen del Patriarcado". En SÁNchez Romero, M. (ed.): Arqueología y género. Granada: UGR, pp. 73-108.

Hernando, A. (2007): "Sexo, Género y Poder. Breve reflexión sobre algunos conceptos manejados en la $\mathrm{Ar}-$ queología del Género", Complutum, 18, pp. 167-174.

Hernando, A (2012): La fantasía de la individualidad. Sobre la construcción sociohistórica del sujeto moderno. Buenos Aires: Katz.

Hodder, I. (2012): Entangled. An archaeology of the relationships between Humans and Things. Oxford: Willey-Blackwell.

Lemonnier, P. (1993): Introduction to Technological choices: Transformation in Material Cultures since the Neolithic. London: Routledge.

Lemonnier, P. (2012): Mundane Objects: Materiality and Non-verbal Communication. Walnut Creek, California: Left Coast Press.

Livingstone, A. (2007): Chaîne Operatorie de la Poterie. Réferénces ethnografiques, analices et reconstitution. Tervueren: Musée Royal de l'Afrique Centrale.

Longacre, W. A. (1999): "Standardization and Specialization: What's the Link?”. En Sкiво, J. M. y Feinman, G. M. (eds.): Pottery and People. Salt Lake City: UUP, pp. 44-58.

Luezas, R. A. y Martín Bueno, M. (1995): "Cerámica pintada romana de tradición indígena procedente de Bilbilis (Calatayud, Zaragoza)", Espacio, Tiempo y Forma. Serie I. Prehistoria y Arqueología, 8, pp. 235293.

Mañas, I. y Bermejo, J. (2013): "El debate en torno al crecimiento económico en el mundo romano: una propuesta analítica a partir de la arqueología de las unidades domésticas", Gerión, 31, pp. 269-286.

Mariné, M. y Ruiz Zapatero, G. (1988): "Nuevas investigaciones en Las Cogotas. Una aplicación del 1\% cultural", Revista de Arqueología, 84, pp. 46-53.

Morel, J. P. (1991): "El artesano". En Giardina, A.; Castaño, J.; Matesanz, J. y Quesada, F. (eds.): $E l$ hombre romano. Madrid: Alianza Edit., pp. 259-288.

Nicholson, P. T. (2009): "Pottery Production". En Encyclopedia of Egiptology. Los Ángeles, California: UCLA, pp. 1-8.

Padilla, J. J. (2011): "El alfar de Las Cogotas (Cardeñosa, Ávila): Una mirada etnoarqueológica y experimental", Arqueología y Territorio del Dpto. de Prehistoria y Arqueología de la Univ. de Granada, 8, pp. 115-128.

Padilla, J. J. y Chapon, L. (2015): "Gender and Childhood in the II Iron Age: the pottery centre of Las Cogotas (Ávila, Spain)". En Sánchez Romero, M.; Alarcón, E. y Aranda, G. (eds.): Children, Spaces and Identity. Oxford y Filadelfia: Oxbow, pp. 75-87.

Peña, J. T. (2007): Roman Pottery in the archaeological record. Cambridge: cup.

Perlines, M. R. (2005): "La presencia de cerámica a Torno en contextos anteriores al cambio de milenio. Propuesta para su estudio". En Celestino, S. y Jiménez Ávila, J. (eds.): El Periodo Orientalizante. Actas III Simposio Internacional de Arqueología de Mérida: Protohistoria del Mediterráneo Occidental. Mérida-Madrid: csic, pp. 477-489.

Pikirayi, I. y Lindahl, A. (2013): "Ceramics, Ethnohistory, and Ethnography: Locating Meaning in Southern African Iron Age Ceramic Assemblages", African Archaeological Review, 30 (4), pp. 455-473.

Polo, J. (1999): "Las cerámicas pintadas romanas de tradición indígena: aportaciones estratigráficas de la ciudad hispano romana de Complutum". En Bueno, P. y De Balbín, R. (coords.): Actas II Congreso de Arqueología Peninsular. Zamora: frae, vol. 4, pp. 89-102.

Quinn, P. S. (2013): Ceramic Petrography. Oxford: Archaeopress.

Río-Miranda, J. (2017): "La cerámica pintada romana de tradición indígena en Cáparra-Extremadura", Cuadernos Caparenses, 16, pp. 1-50. 
Roca, M.; Buxeda, J. y Madrid, M. (2014): "El centro de producción de Terra Sigillata Hispánica altoimperial de Andújar. Nuevos datos y algunas puntualizaciones a partir del estudio de un lote de moldes". En Roca, M.; Madrid, M. y Celis, R. (eds.): Contextos cerámicos de época altoimperial en el Mediterrráneo Occidental. Barcelona: UB, pp. 429-481.

Rodríguez González, E. (2012): "La transición del s. vi al v a. C. en el bajo Guadalquivir: La transformación de los repertorios cerámicos". En CaSCALHeIRA, J. y Gonçalves, C. (eds.): Actas das IV Jornadas de Jovens em Investigação Arqueológica. Promontoria Monográfica, 16 (2). Faro, pp. 239-246.

Rodríguez Neila, J. F. (2014): "Trabajo, identidad social y estatus jurídico de los artesanos en el ámbito urbano de Hispania". En Bustamante, M. y BerNaL, D. (eds.): Artífices idóneos. Artesanos, talleres y manufacturas en Hispania. Mérida: csic, pp. 13-42.

Roux, V. (2003): “A Dynamic Systems Framework for Studying Technological Change: Application to the Emergence of the Potter's Wheel in the Southern Levant", Journal of Archaeological Method and Theory, 10 (1), pp. 1-30.

Roux, V. (2008): "Evolutionary trajectories of technological traits and cultural transmission: a qualitative approach to the emergence and disappearance of the ceramic wheel-fashioning technique in the Southern Levant during the fifth to third millennia BC". En Stark, B.; Bowser, B. y Horne, L. (eds.): Cultural Transmission and Material Culture. Breaking Down Boundaries. Tucson: Aup, pp. 82-104.

Roux, V. (2009): "Technological innovations and developmental trajectories: social factors as evolutionary forces. Innovation in cultural systems". En O’Brien, M. y Shennan, S. (eds.): Contributions from evolutionary anthropology. Cambridge: MIT Press, pp. 217-234.

Roux, V. y Corbeta, D. (1990): Le tour du potier. Spécialization artisanale et compétences techniques. Coll. Monographies du CRA, 4. Paris: CNRS.

Ruiz Gálvez, M. L. (2013): "Almudena Hernando Gonzalo. La fantasía de la individualidad. Sobre la construcción sociohistórica del sujeto moderno. Katz Conocimiento. 2012", Complutum, 24 (1), pp. 223-225.

Ruiz Montes, P. (2011): "Bases para un estudio socioeconómico de las aglomeraciones productivas en el mundo romano, elementos para una aproximación a dicho fenómeno en el Alto Guadalquivir (ss. I-II d. C.)", Cuadernos de Prehistoria y Arqueología de la Univ. de Granada, 21, pp. 303-322.
Ruiz Montes, P. (2014): Romanización y producción de cerámicas finas en las áreas periféricas de la provincia Bética. Factores de implantación, comercio y desarrollo técnico en el suburbium artesanal de Isturgi Triumphale (Los Villares de Andújar, Jaén) (ss. I-II d.n.e.). BAR Intern. Ser., 2442. Oxford: Archaeopress.

Ruiz Valderas, E. (1988): "Cerámica pintada de tradición indígena en la Cartagena romana”. En Arte y poblamiento en el SE peninsular. Antigüedad y Cristianismo, v. Murcia, p. 621.

SaÉnz Preciado, J. C. (1999): "Aportaciones a la Terra Sigillata hispánica en Aragón”. En Balbín, R. y BueNo, P. (eds.): II Congreso de Arqueología Peninsular. Arqueología romana y medieval. Alcalá de Henares, pp. 159-169.

Sáenz Preciado, J. C. (2014): "La Sigillata Hispánica ¿Artesanía o manufactura?”. En Martín Bueno, M. y Sáenz Preciado, J. C. (eds.): Modelos edilicios y prototipos en la monumentalización de las ciudades de Hispania. Monografías Arqueológicas. Arqueología, 49. Zaragoza, pp. 149-162.

SÁez Romero, A. M. (2011): "El alfar tardopúnico de Torre Alta. Balance y estado de la cuestión tras veinte años de investigaciones". En Díaz Rodríguez, J. J.; Sáez Romero, A. M.; Vijande, E. y Lagóstena, J. (eds.): Estudios recientes de Arqueología Gaditana. Actas Jornadas de Jóvenes Investigadores. Prehistoria y Arqueología (Cádiz, 2008). Bar Intern. Ser., 2276. Oxford: Archaeopress, pp. 183-202.

Saiz Carrasco, M. ${ }^{a}$ E. (2005): "Propuesta de estudio a aplicar en los alfares celtibéricos del Sistema Ibérico Central", Salduie, 5, pp. 113-130.

Saiz Carrasco, M. ${ }^{a}$ E. y Gómez Villahermosa, S. (2008-2009): "Avance del estudio de la alfarería celtibérica en la Oruña (Vera de Moncayo-Trasmoz, Zaragoza)", Turiaso, 19, pp. 34-61.

Sánchez López, E. y Gozalbes, E. (2012): "Los usos del agua en la Hispania Romana", Vinculos de Historia, 1 , pp. 11-29.

Sánchez Simón, M. (1995): "Notas sobre la cerámica pintada de tradición indígena a comienzos de la época flavia en Uxama (Osma, Soria)", Boletín del Seminario de Estudios de Arte y Arqueología, 61, pp. 125-144.

SChiffer, M. B. (1992): Technological perspectives on behavioural change. Tucson: UAP.

Sсотт, J. C. (2000): Los dominados y el arte de la resistencia. México: Era.

Sempere, E. (1992): "Catalogación de los hornos de España y Portugal”. En Tecnología de la cocción cerámica 
desde la antigüedad a nuestros días. Alicante: Museo de Alfarería de Agost, pp. 185-237.

Skibo, J. M. y Schiffer, M. B. (2008): A Behavioural Approach to Material Culture. New York: Springer.

Sotomayor, M. (1970): "Siete hornos de cerámica romana en Granada con producción de sigillata". En Actas XI Congreso Nacional de Arqueología (Mérida, 1968). Zaragoza, pp. 713-728.

Taracena, B. (1931-32): "La cerámica de Clunia", Archivo de Prehistoria Madrileña, II-III, pp. 85-91.

Thér, R.; Mangel, T. y Gregor, M. (2017): “Potter's wheel in the Iron Age in Central Europe: process or product innovation?", Journal of Archaeological Method and Theory, 24 (1), pp. 1-44.

Tremoleda, J. y Castanyer, P. (2013): "El alfar romano de Ermedàs. El taller y su producción (Cornellà del Terri, Girona)". En Bernal, D.; Juan Tovar, L. C.; Bustamante, M.; Díaz Rodríguez, J. J. y SÁez, A. M. (eds.): Hornos, talleres y focos de producción alfarera en Hispania. Monografías Ex Officina Hispana, 1. Cádiz: UCA, pp. 251-256.

Tsiolis, V. G. (1997): "Las restricciones de la producción tegularia en la Lex Ursonensis", Studia Historica. Historia Antigua, 15, pp. 119-136.

Vallejo, J. I. (2007): "Cambio tecnológico en la producción de cerámica protohistórica de la península ibérica: La introducción del torno de alfarero". En Serra, F. (ed.): Mediterranea. Roma: Quaderni di Archeologia Etrusco-Italica, pp. 9-32.

VAN DeR Leeuw, S. E. (1993): "Giving the potter a choice. Conceptual aspects of potterytechniques". En LEmonnier, P. (ed.): Technological choice. Transformations in material cultures since the Neolitic. London: Routledge, pp. 238-288.

Velde, B. y Druc, I. C. (1999): Archeological ceramic materials origin and utilization. Natural science in archaeology. Berlin-Heidelberg: Springer. 